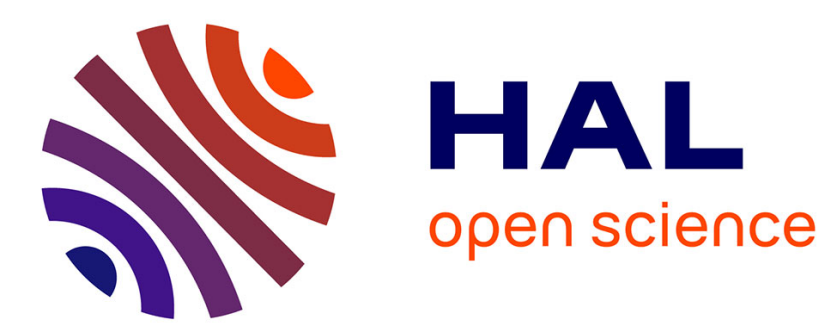

\title{
Evaluation of a new Radio Technology and Visible Light Communication for a Platooning Application
}

\author{
Fouzi Boukhalfa, Mohamed Hadded, Paul Mühlethaler, Oyunchimeg Shagdar
}

\section{To cite this version:}

Fouzi Boukhalfa, Mohamed Hadded, Paul Mühlethaler, Oyunchimeg Shagdar. Evaluation of a new Radio Technology and Visible Light Communication for a Platooning Application. ISNCC 2021 International Symposium on Networks, Computers and Communications, Oct 2021, Dubai, United Arab Emirates. hal-03364383

\section{HAL Id: hal-03364383 https://hal.science/hal-03364383}

Submitted on 4 Oct 2021

HAL is a multi-disciplinary open access archive for the deposit and dissemination of scientific research documents, whether they are published or not. The documents may come from teaching and research institutions in France or abroad, or from public or private research centers.
L'archive ouverte pluridisciplinaire HAL, est destinée au dépôt et à la diffusion de documents scientifiques de niveau recherche, publiés ou non, émanant des établissements d'enseignement et de recherche français ou étrangers, des laboratoires publics ou privés. 


\title{
Evaluation of a new Radio Technology and Visible Light Communication for a Platooning Application
}

\author{
Fouzi Boukhalfa*, Mohamed Hadded*, Paul Muhlethaler ${ }^{\dagger}$ and Oyunchimeg Shagdar* \\ *Institute VEDECOM, 23 bis allée des Marronniers, 78000 Versailles, France \\ ${ }^{\ddagger}$ EVA Team, Inria Paris, 2 Rue Simone Iff, 75012 Paris, France
}

\begin{abstract}
The autonomous platoon is today one of the key tools for better road utilization. In fact, by optimizing the distance between vehicles, the air drag is reduced, and researchers have shown that $20 \%$ of energy can be saved by using this concept. From a network point of view, reducing the distance between vehicles will allow a new point-to-point communication link between the vehicles in front and behind by using Vehicular Visible Light Communication (V-VLC), thus providing an opportunity to have a hybrid communication. Our new radio design based on the AS-DTMAC protocol guarantees a high Quality of Service for real-time applications. However, with a very high density, we can reach the bandwidth dedicated to $\mathrm{V} 2 \mathrm{X}$ radio. In the case of a platoon, this scenario can cause dangerous platoon instability. Assisting the radio with another communication vector such as V-VLC can help to maintain the high level of reliability that is necessary for the control of a platoon. In this paper, we first carry out an analytical analysis to investigate the capacity of our new radio technology to support the platoon control use case in terms of the quality of service $(\mathrm{QoS})$ required for this type of application. Secondly, we show through extensive simulations the current level of $\mathrm{V}$ VLC technology, compared to radio technology, in terms of packet loss and delay.

keywords - Autonomous vehicles, platooning, network simulation, OMNET, VEINS-VLC framework.
\end{abstract}

\section{Introduction and motivation}

Nowadays, our highways are becoming increasingly congested. For the best traveling experience, Intelligent Transportation Systems (ITS) aim to optimize road utilization and traffic management. To address congestion issues, conventional vehicle systems for traffic flow like the Adaptive Cruise Control (ACC) are not sufficient. This system automatically adjusts a vehicle's speed based on information from vehicles ahead. A more dynamic regulation strategy requires a cooperative system which will provide more information on the positions, speeds and accelerations of neighboring vehicles. Thanks to these cooperative systems, new vehicular applications can be established (an example of such a system being Cooperative Adaptive Cruise Control (CACC)). Platooning is one of the best-known applications that can enhance road capacity. A Platooning application involves a robust control with an efficient communication strategy. Due to high QoS requirements in terms of update frequency (at least $10 \mathrm{~Hz}$ ) with a recommended transmission latency of $20 \mathrm{~ms}$ and a reliability of $99.99 \%$, researchers are looking to combine more than one communication technology. In the literature, many communication strategies have been proposed. To achieve low-latency and ultra-reliable communication, an additional vector of communication is required since relying only on the Radio Frequency (RF) link, as we developed in [1] [2] [3] [4] [5], may not be sufficient. With a very high number of nodes, there is a risk of reaching the full capacity of the channel and, if that occurs, some nodes will be unable to communicate with the rest of the network. In such a case, Vehicular Visible Light Communication (V-VLC) is a potential technology that could assist RF communication. However, V-VLC in outdoor environments faces a number of challenges due to mobility, weather conditions and ambient light [6]. Nonetheless, it provides a large free spectrum at a relatively low cost as the Light Emitting Diodes (LEDs) are already embedded in modern vehicular light systems [6].

Our goal is twofold: first, to show that our new RF design is able to support platooning applications (to our best knowledge, we are the first to propose a radio technology based on a fully distributed TDMA which meets the QoS requirements necessary for platooning communication). Secondly, we set out to test the maturity of the V-VLC standard in a large-scale simulations for dynamic V-VLC scenarios such as platooning in the presence of interfering vehicles. For this, we have chosen to use a simulator based on a fairly realistic empirical model that takes into account the impact of the type of vehicle (the features of this framework are explained below). This study helps us to determine whether light links are sufficiently stable to be used in place of radio links, or whether we should use both communication links to improve the overall reliability.

The remainder of this paper is organized as follows. In the next section, we present related work. In Section 3, we discuss and prove analytically the capacity of our new radio design to support a platooning application. For this task, we select suitable metrics, namely average access time and path loss (under NLOS conditions). In Section 4, we show the performance of the V-VLC link in the presence of disturbing vehicles. We evaluate the PDR for various network conditions, different headlight modules (provided by Veins VLC [7] [8]) and speeds. We also compare the 
performance between RF and VLC in terms of PDR and delay. Finally, in Section 5, we conclude the paper outline future work..

\section{Related work}

The IEEE 802.15.7 [16], published in 2011, is the current Standard for Short-Range Optical Wireless Communications and includes outdoor communications. However, this standard is not specially designed for V-VLC and there is no effort being made in this direction: the standardisation efforts mainly focus on the system costs. Recent research has shown the need for a dedicated new standard for vehicular networks [17].

Light communications are classified into two categories depending on the environment: indoor (mainly using LiFi applications [10][11]), and outdoor (to establish communication between vehicles and the infrastructure [9]). The VLC wavelength varies from $380 \mathrm{~nm}$ to $780 \mathrm{~nm}$ of the electromagnetic spectrum. VLC will benefit from a large available spectrum, which will result in high data rates. A VLC receiver can encompass either a Photodiode (PD) or a camera. The location of a vehicle's LED-based light makes the set-up of a full-duplex communication possible (see Figure 1). A vehicle has two tail-lights and two head-lights. Sunlight and outside light sources can cause, respectively, a shot noise and interference. V-VLC requires a line-ofsight (LOS) scenario but some research shows that it is possible to use ground reflections to have V-VLC NLOS communication [12]. However, the LOS scenario is not always available due to a variations in the vehicle's heading or to weather conditions.

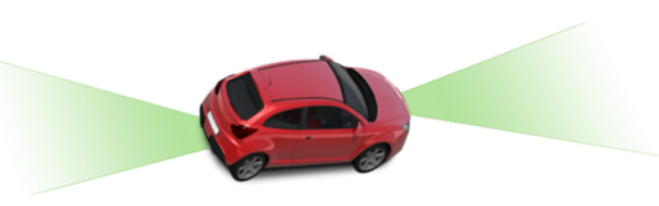

Figure 1. Vehicle LED-based light

Due to the propagation characteristics of light (see Figure 2), which can not pass through objects, NLOS messages need to be forwarded in a multi-hop manner. VLC technology can provide an alternative for GPS-based positioning technology, which is not always available (e.g. in a tunnel or an indoor environment). The precision of such a system is about a few centimeters, whereas GPS gives an error of $10 \mathrm{~m}$ [9]. VLC uses multi-hop communication to reach vehicles that are not directly in front, resulting in high latency, whereas RF can reach all these vehicles immediately. On the other hand, in high density scenarios, V-VLC has good scalability compared to RF. If the RF band is restricted or unavailable (due to safety or military applications,) using VLC would be a suitable alternative.

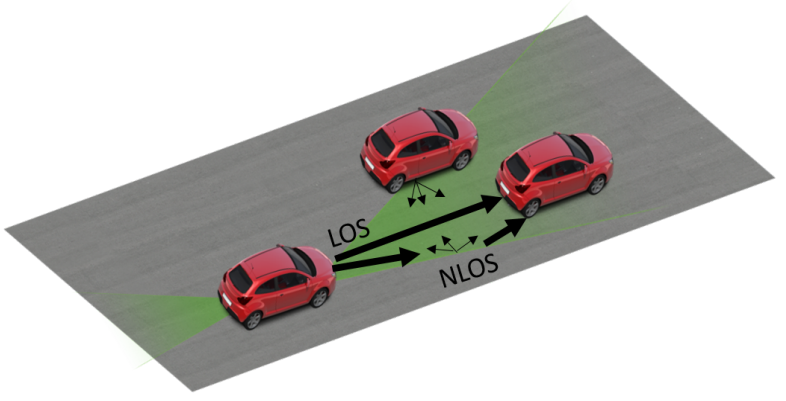

Figure 2. A LOS \& NLOS V-VLC scenario

A Platooning application involves a robust control with an efficient communication strategy. As mentioned above, due to high QoS requirements in terms of update frequency (at least $10 \mathrm{~Hz}$ ) with a recommended transmission latency of $20 \mathrm{~ms}$ [13][14], and a reliability of $99.99 \%$, research tends to combine more than one communication technology. In the literature, different communication strategies have been proposed. Figure 3 and Figure 4 summarize the main communication strategies.

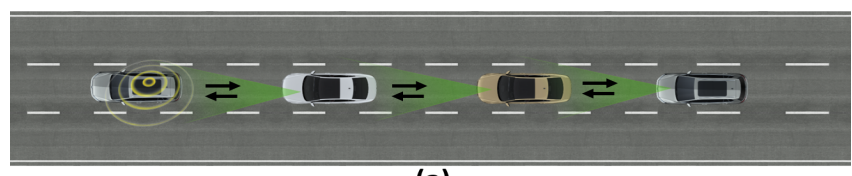

(a)

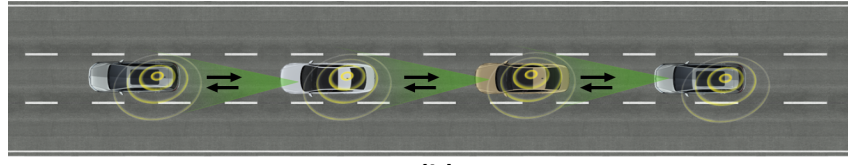

(b)

Figure 3. Communication strategy based on RF-VLC

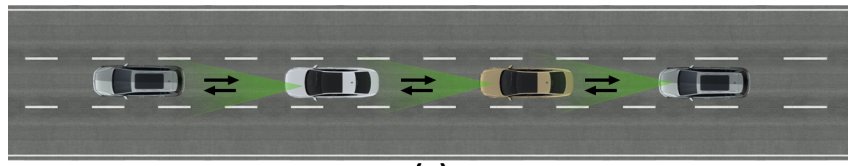

(a)

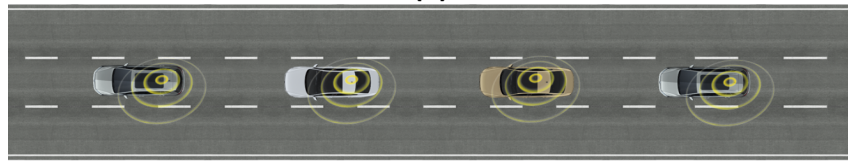

(b)

Figure 4. Communication strategy based on one communication technology 
In Figure 3 (a) each platoon member is using V-VLC. Only the leader is using both V-VLC and RF to communicate with its followers. In Figure 3 (b) the platoon members are in redundant mode (both RF and V-VLC are being used). In Figure 4 (a) and (b) the vehicles are using only one vector at a time, either V-VLC or RF. Such an example can be found in [15] where Segata et al. propose IEEE 802.11p/IEEE 1609.4 PHY/MAC to manage communication between the vehicle members of a platoon, They assume that each vehicle has the capacity to know its position in the platoon. The leader's beacon is used as a synchronisation signal to divide the time into slots. The number of slots depends on the size of the platoon, and the allocation of slots to vehicles will depend on their position with respect to the leader. The idea is to adapt the transmission power: the leader will use enough transmission power to reach each follower, whereas the followers will adapt their power to reach only those vehicles that require such data. This will reduce interference with other vehicles and increase spatial reuse.

\section{Performance Analysis of AS-DTMAC for platooning control}

Following the AS-DTMAC scheme described in previous work [1][2][3][4][5], a vehicle loses its slot when it changes zones (see Figure 5: $\mathrm{R}$ equals the transmission range). Thanks to the active signaling mechanism, reserving another slot in the new area will take only a few milliseconds. Now, considering a platooning scenario, the members of the platoon are considered as a single entity. Therefore, in order to maintain communication between the leader and the followers, all platoon members must be allocated a slot in less than $100 \mathrm{~ms}$ (platoon update rate), otherwise, data are lost and this can affect the platoon's stability.

\section{Platoon scenario with RF based on AS-DTMAC}

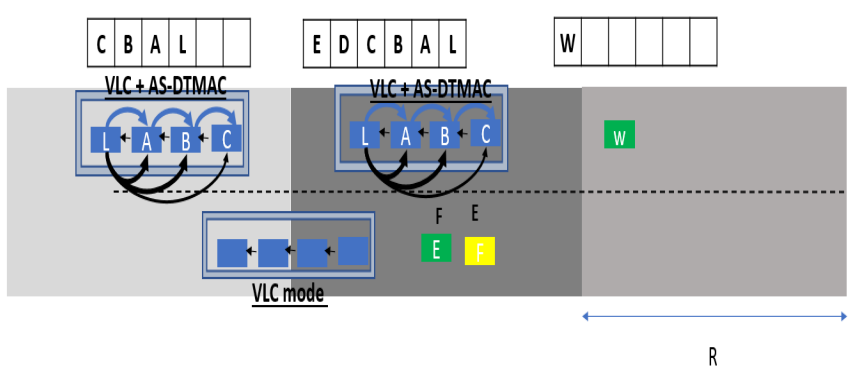

Figure 5. Platoon scenario with RF based on AS-DTMAC

We assume that we have $n$ vehicles in the platoon and we wish to compute the average time needed for all the members of the platoon to obtain a slot in the new area, thus in a new DTMAC frame. We assume that the slots in the new areas are occupied with probability $\lambda$ and free with probability $1-\lambda^{1}$. We also assume that the free slots are

1. This is a simplified model for AS-DTMAC which assumes that the DTMAC frame is infinite obtained by AS-DTMAC in a greedy mode (the first slot available is reserved by a vehicle with AS-DTMAC without any collision since the collision rate is extremely small). We note by

$I_{1}+1, I_{1}+I_{2}+2, \ldots, I_{1}+I_{2}+\cdots+I_{n}+n$ the slots successfully obtained by the vehicles in the platoon. The probability that $I_{1}=i_{1}, I_{2}=i_{2}, \ldots I_{n}=i_{n}$ is

$$
(1-\lambda) \lambda^{i_{1}}(1-\lambda) \lambda^{i_{2}} \ldots(1-\lambda) \lambda^{i_{n}}
$$

and the total time needed for all the nodes to obtain a slot in the next frame is $n+I_{1}+I_{2}+\cdots+I_{n}$.

The average time required for all the nodes to obtain a slot is thus

$$
E\left(\left(1+I_{1}\right)+\left(1+I_{2}\right)+\cdots+\left(1+I_{n-1}\right)+\left(1+I_{n}\right)\right)
$$

where $E$ means the expectation on all the possible events.

But all the $I_{j}$ with $j \in 1,2, \ldots, n$ are independent with the same law and we have:

$$
E\left(\left(1+I_{1}\right)+\cdots+\left(1+I_{n}\right)\right)=n E\left(1+I_{1}\right)=\frac{n}{1-\lambda} .
$$

We also have

$P\left(\left(n+I_{1}+\cdots+I_{n}=n+p\right)=\left(\begin{array}{c}n+p-1 \\ n-1\end{array}\right) \lambda^{p}(1-\lambda)^{n}\right.$.

We have to notice that the last slot must be free, this explains the presence of the -1 in the formula. We can easily compute the distribution of $n+I_{1}+\cdots+I_{n}$.

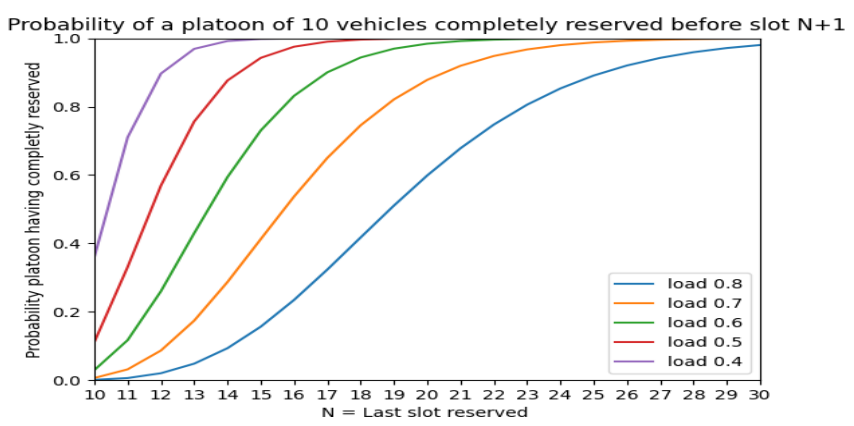

Figure 6. Probability of a platoon of 10 vehicles completely reserved before slot $\mathrm{N}+1$

In Figure 6, we plot the probability that the last slot successfully reserved by a platoon of 10 vehicles is at most slot number $N$. We observe that unless there is a high load, the reservation stops before the end of the AS-DTMAC frame whose duration is 33 slots.

In order to further investigate the capacity of our new radio technology design to support the platoon application, we analyze the connectivity between platoon members in fading channel conditions, and under our active signaling mechanism.

V2X applications operate on the $5.9 \mathrm{GHz}$ bandwidth, and the platooning application requires a safety distance $(d)$ between platoon members of between $10 \mathrm{~m}$ and $30 \mathrm{~m}$. We can consider that $d>>\lambda(\lambda$ here refers to the radio 
wave length) and thus we use statistical models to take into account the fading effect (including the random channel gain and random phase shift). The following formula gives the path loss with channel fading:

$$
P L(d)=10 * \gamma * \log \left(d / d_{0}\right)+P L_{0}\left(d_{0}\right)+X
$$

$X$ is a zero mean complex normal distribution variable with standard deviation $\sigma$. The $\gamma$ is the path loss exponent. The values of $\sigma$ and $\gamma$ were taken from [19]. AS-DTMAC uses the physical layer of the IEEE 802.11 p thus the RX sensitivity is $-85 \mathrm{dbm}$ (64-QAM with $3 / 4$ coding) [18]. In a previous paper [3] The following results were obtained by using the Monte-Carlo simulation.

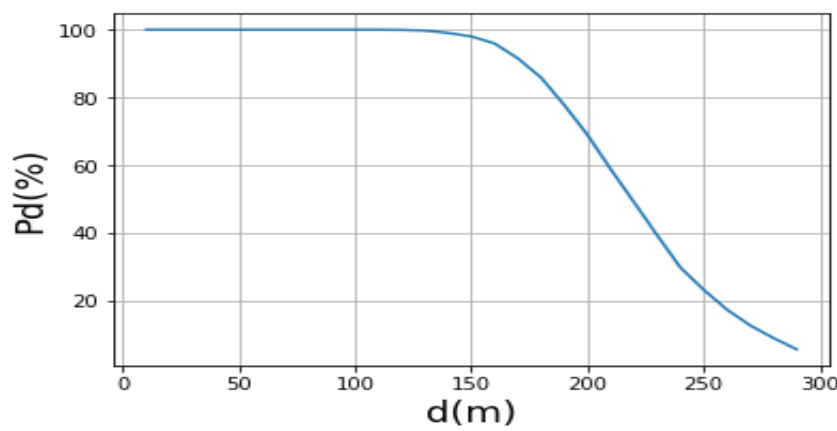

Figure 7. Probability of detecting \& decoding versus RX-TX vehicle interdistance

Figure 7 shows the probability of correctly detecting and decoding $\left(P_{d}\right)$ a packet under fading conditions versus the distance between the transmitter and the receiver. From this curve we can see that up to $150 \mathrm{~m}, 100 \%$ of packets are received correctly. Beyond this value, the $P_{d}$ gradually decreases by increasing the distance. In Figure 8 , we vary the variance $(\sigma)$ of the fading (the sweep values were taken from the reference [20]) and show their impact on $P_{d}$. we plot four curves, each one corresponding to a particular distance $(150 \mathrm{~m}, 170 \mathrm{~m}, 190 \mathrm{~m}, 210 \mathrm{~m})$. The results show that there is an impact of this parameter on the detection: by raising $\sigma$, the probability decreases; however, the probability decreases more rapidly when the distance grows. Finally, in Figure 9 we vary the path loss exponent $(\gamma)$ from 2.2 to 3 [20]. As we can observe, this last parameter also has an impact on $P_{d}$. Furthermore, for each curve plotted, we can see that the threshold to have $100 \%$ of packets moves with the distance (i.e. the greater the distance is, the more the threshold moves to the left).

From this connectivity analysis, we can conclude that safe connectivity can be achieved at a distance of $150 \mathrm{~m}$. However, the above study (i.e. the probability of a platoon having a completely reserved slot) shows that regarding the AS-DTMAC functionality we limit the platoon size to 10 vehicles with an assumed inter-vehicle distance of $10 \mathrm{~m}$ and a mean vehicle length of 4-5 m.

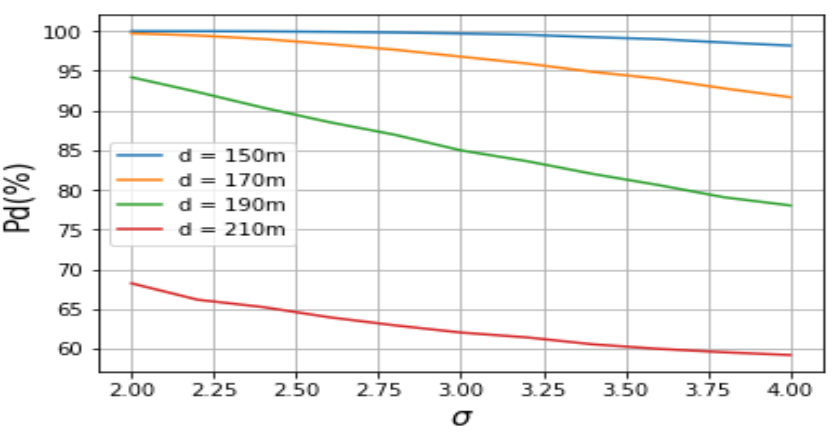

Figure 8. Probability of detecting \& decoding versus Rayleigh-fading variance $\sigma$

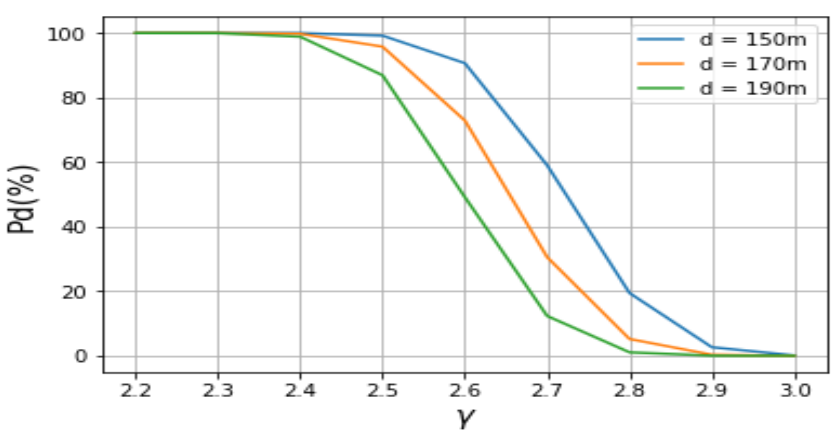

Figure 9. Probability of detecting \& decoding versus the path loss exponen

\section{Simulation results}

The simulation tool for V-VLC used in this section is based on VEINS-VLC. This simulator uses an empirical model (i.e. it uses photometric data and thus takes into account the NLOS component coming from the ground). As the data are collected from a real headlight module, this radiation pattern includes the effect of a high and low beam $^{2}$ in the headlight coming from real vehicular headlight modules. These modules are from HELLA GmbH \& Co. KGaA [7][8]. Figure 10 summarizes the integration of the empirical model into the VEINS framework (for more details see [8]).

TABLE 1. Simulation parameters

\begin{tabular}{cc}
\hline Parameter & Value \\
\hline PacketBytelength & $1024 b y t e$ \\
BeaconingFrequency & $10 \mathrm{~Hz}$ \\
Channel model & veins-vlc \\
Car-Following-Model & $\mathrm{CACC}$ \\
Bitrate & $1 \mathrm{Mbps}$ \\
Disturbing vehicles speed & $50-120 \mathrm{~km} / \mathrm{h}$ \\
\hline
\end{tabular}

2. The low beam in the headlight is to implement an asymmetric light distribution. 


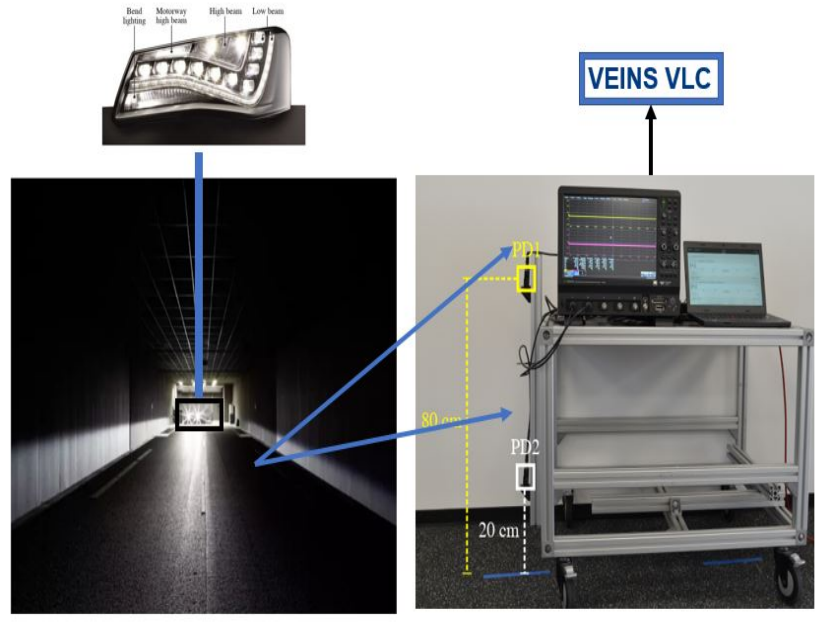

Figure 10. Summary of the integration of the empirical model in VEINSVLC (the images were taken from [8]): the measurements were done at the HELLA's Lichtkanal company in a light channel room of dimension $145 \mathrm{~m} * 11 \mathrm{~m}$. For the emissions, a real pair of headlights module was used, whereas the receiver side uses two Photodiodes (PDs) of type PDA100A placed at the same level as the license plate and the tail lights.

\subsection{V-VLC performance with presence of interfer- ence}

In this section, we show the impact of interference on the performance of visible light communication in a vehicular platoon. Our study focuses on the vehicles that are arriving in parallel to the platoon's lane. When there is packet in the channel transmitted between the platoon vehicles, the channel is disturbed by the light of these arriving vehicles. This will continue to be be the case until all the disturbing vehicles have overtaken the platoon (see Figure 11).

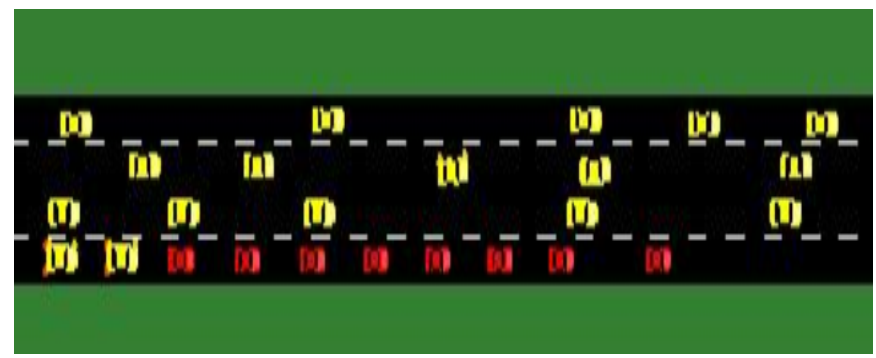

Figure 11. A highway scenario with a platoon in the lower lane, the other lanes are for connecting vehicles.

Figure 12 shows the Packet Delivery Ratio (PDR) versus the number of disturbing vehicles. We plot different curves, each one using a particular model available on the market (LbSedan1, LbSedan2, Lbsuv1, HbSedan2, HbSedan1). All the curves decrease as a function of the number of disturbing vehicles. As we can observe, the interference caused by the vehicles' lights has an important impact, particularly for high numbers of disturbing vehicles (for 100 disturbing vehicles: $40 \%$ of the packets are lost). We also see that the types of vehicles in platoon impact the PDR and the difference can reach $10 \%$ for high densities.

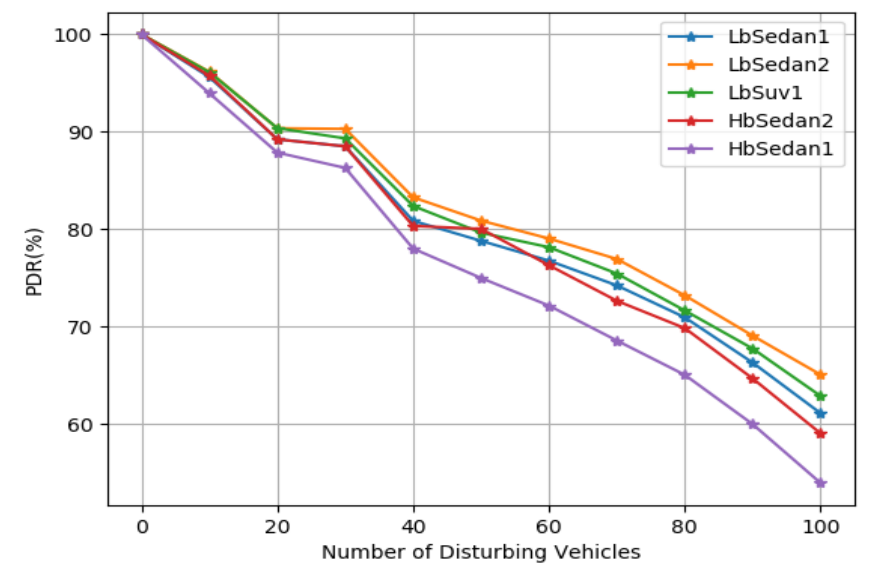

Figure 12. V-VLC PDR versus number of disturbing vehicles: Simulation time $=116 \mathrm{~s} ;$ platoon size $=8 ;$ platoon inter-vehicle distance $=10 \mathrm{~m}$; Disturbing vehicles' speed $=100 \mathrm{~km} / \mathrm{h} ;$ Platoon vehicles' speed $=15$ $\mathrm{km} / \mathrm{h}$.

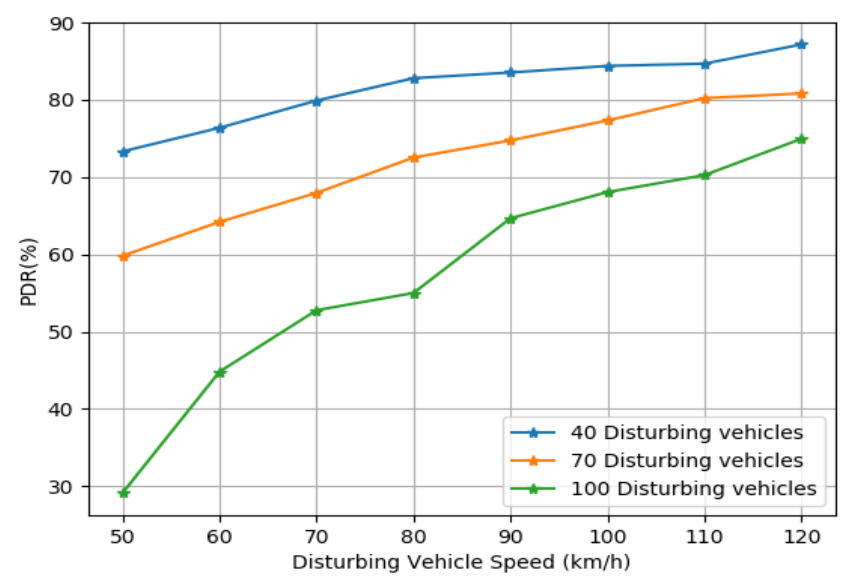

Figure 13. V-VLC PDR versus disturbing vehicle speed: Simulation time $=208 \mathrm{~s}$; platoon size $=8$; platoon inter-vehicle distance $=10 \mathrm{~m}$; Disturbing vehicles' speed $=100 \mathrm{~km} / \mathrm{h}$; Platoon vehicles' speed $=15 \mathrm{~km} / \mathrm{h}$; Headlight module = "HbSedan2".

Figure 13 shows the impact of the speed of disturbing vehicles on the PDR. We vary the speed between $50 \mathrm{~km} / \mathrm{h}$ to $120 \mathrm{~km} / \mathrm{h}$, and we plot three curves for 40,70 , and 100 disturbing vehicles respectively. The results show that the PDR is better when the speed increases. In fact, the greater the speed of the disturbing vehicles, the shorter the interference time is, which results in a better PDR.

Figure 14 shows the impact of platoon vehicle interdistance on the PDR. We vary the distance between $10 \mathrm{~m}$ to $40 \mathrm{~m}$, and we plot three curves for 40,70 , and 100 


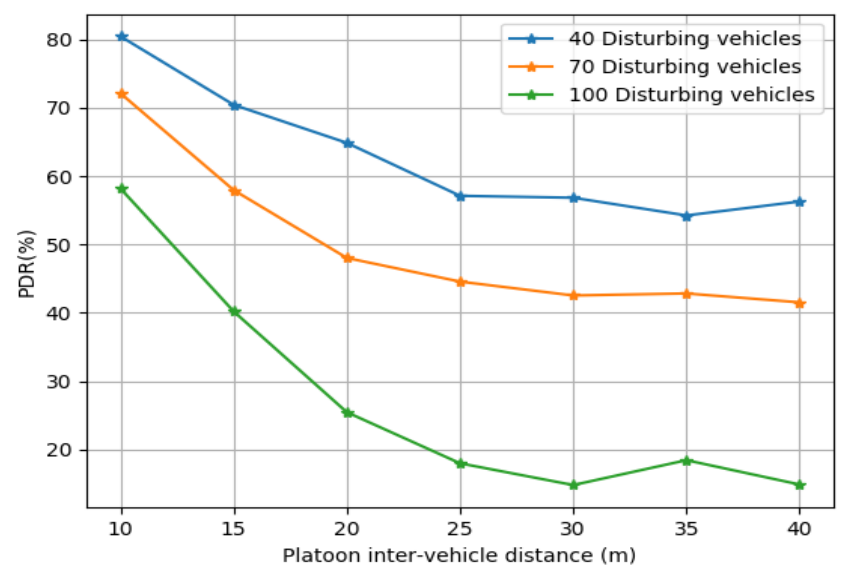

Figure 14. V-VLC PDR versus platooning vehicle inter-distance: Simulation time $=116 \mathrm{~s}$; platoon size $=8$; Platoon vehicles' speed $=15 \mathrm{~km} / \mathrm{h}$; Disturbing vehicles' speed $=100 \mathrm{~km} / \mathrm{h}$; Headlight module $=$ "HbSedan2".

disturbing vehicles respectively. The results show that the PDR is better when the communication is under $10 \mathrm{~m}$. In fact, beyond $10 \mathrm{~m}$, the PDR decreases rapidly until reaching a communication distance of $25 \mathrm{~m}$, then the PDR becomes more stable for all curves.

\subsection{Performance comparision between V-VLC and RF}

In this section, we discuss and compare the performance of RF and V-VLC in terms of PDR and delays. We consider the same scenario as in Figure 11 extended by a parallel highway for vehicles moving on the opposite side. As concerns VLC propagation, only the two lanes near the platoon lane will have an impact on the performance, the rest will have no impact, whatever the density is. As described before, AS-DTMAC divides the road into different zones, and each zone has a set of slots (about 33 slots per zone), thus, the performance of the RF can be impacted when the number of vehicles rises since more vehicles are reserving a slot and there is a risk of reaching the total resource capacity.

Figure 15 shows the delay for V-VLC versus platoon beacon packet length. Obviously, the delay increases as the packet length increases. The delay varies from $1 \mathrm{~ms}$ to $9 \mathrm{~ms}$ for packets with lengths ranging from 100 to 1200 bytes. Moreover, in a platoon, if, for example, the last member attempts to communicate with the leader via $\mathrm{V}$ VLC (see Figure 11), messages need to be forwarded via multihop communication, which increases the latency. Thus, to respect the QoS requirements of this application, the platoon size must be taken into consideration.

In Figure 16 we plot the PDR versus the number of disturbing vehicle for both RF and VLC. As we can see in this scenario, RF is largely reliable compared to V-VLC. In fact, even for high numbers of disturbing vehicles, RF is not greatly affected (less then $3 \%$ of packets are lost) whereas

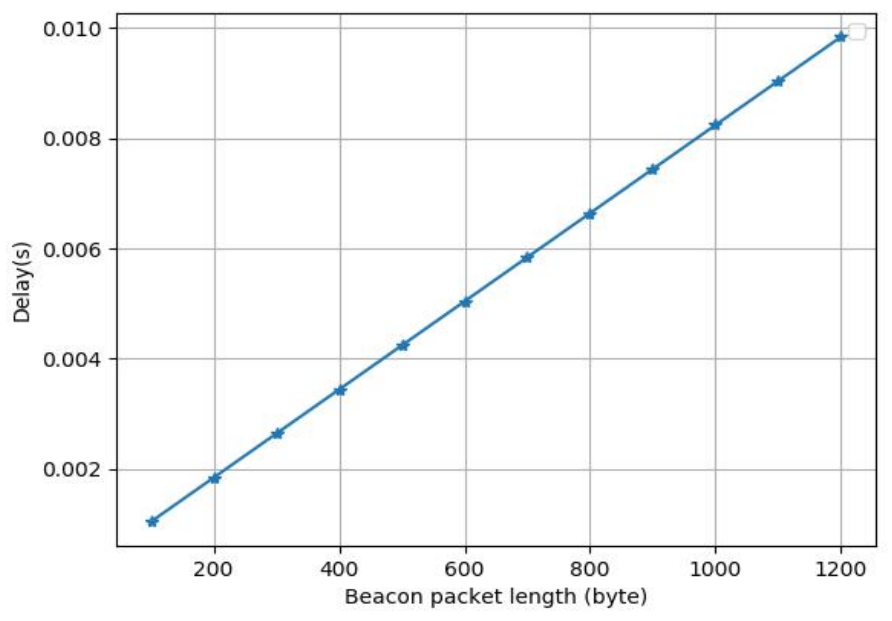

Figure 15. V-VLC delay versus platoon beacon packet length

the PDR for V-VLC decreases more and more (as explained above, this due to light interference).

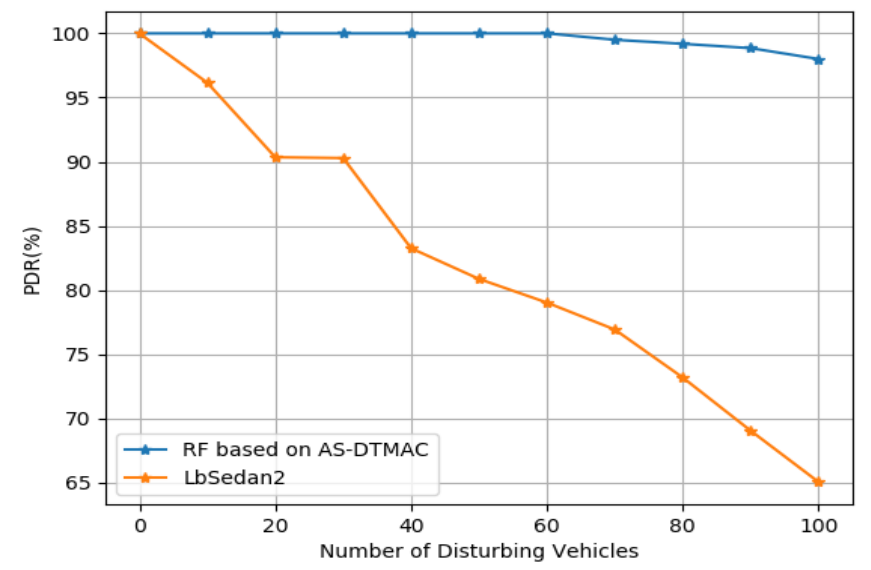

Figure 16. V-VLC and RF PDR versus number of disturbing vehicles

\section{Conclusion}

In this paper, we showed the ability of our radio technology, based on AS-DTMAC, to respond to the QoS requirements of the platooning application. We conducted largescale platoon simulations based on the Veins-vlc framework, which uses a realistic V-VLC model. We also presented the state-of-the-art of V-VLC and showed the lack of maturity of this technology compared to RF. However, V-VLC is still an excellent assistant technology in the platoon use case. By highlighting the limitations of this technology, we will challenge them in the future in order to achieve the full capacity of this technology in terms of data rate, latency and inter vehicular distance. Finally, we compared the performance 
of RF and V-VLC in the platooning scenario in terms of PDR and delay.

In future work, we will focus on integrating RF and VLC communication in a heterogeneous network in order to keep the reliability as high as possible. We plan to propose a smart switching protocol at the handover level to choose the best communication technology depending on the mobility scenario. This protocol will use a dynamic threshold and make decisions based on a vehicular realtime system such as the Channel Busy Ratio (CBR), which gives information about the radio channel quality, but also on V2X by exploiting the information obtained from CAMs to estimate the network load. Thus, the protocol will be able to propose a redundant mode based on the use of RF and V-VLC together in the case when the network load is low.

\section{References}

[1] F. Boukhalfa, M. Hadded, P. Muhlethaler and O. Shagdar, An Active Signaling Mechanism to Reduce Access Collisions in a Distributed TDMA Based MAC Protocol for Vehicular Networks, In International Conference on Advanced Information Networking and Applications, pp. 286-300, Springer, Matsue, Japan, Mar. 2019.

[2] F. Boukhalfa, M. Hadded, P. Muhlethaler and O. Shagdar, An Analytical Model for Performance Analysis of An active Signaling-based MAC Protocol for Vehicular Networks, in Vehicular Technology Conference VTC-FALL, Honolulu, Hawaii, USA, Sept. 2019.

[3] F. Boukhalfa, C. Adjih, P. Muhlethaler, M. Hadded and O. Shagdar, "Physical and MAC Layer Design for Active Signaling Schemes in Vehicular Networks," 2020 16th International Conference on Wireless and Mobile Computing, Networking and Communications (WiMob), Thessaloniki, Greece, 2020, pp. 86-92, doi: 10.1109/WiMob50308.2020.9253440.

[4] F. Boukhalfa, M. Hadded, P. Muhlethaler and O. Shagdar, "Performance Evaluation of an Active Signaling based Time-Slot Scheduling Scheme for connected vehicles;". Annals of Telecommunications annales des télécommunications, Springer, 2020.

[5] F. Boukhalfa, M. Hadded, P. Muhlethaler and O. Shagdar, "Coexistence of IEEE 802.11p and the TDMA-based AS-DTMAC Protocol," 2020 International Conference on Software, Telecommunications and Computer Networks (SoftCOM), Split, Croatia, 2020, pp. 1-6, doi: 10.23919/SoftCOM50211.2020.9238279.

[6] A. Memedi and F. Dressler, Vehicular Visible Light Communications: A Survey, in IEEE Communications Surveys \& Tutorials, doi: 10.1109/COMST.2020.3034224.

[7] Agon Memedi, Hsin-Mu Tsai and Falko Dressler, Impact of Realistic Light Radiation Pattern on Vehicular Visible Light Communication, Proceedings of IEEE Global Communications Conference (GLOBECOM 2017), Singapore, Singapore, December 2017.
[8] Agon Memedi, Claas Tebruegge, Julien Jahneke and Falko Dressler, "Impact of Vehicle Type and Headlight Characteristics on Vehicular VLC Performance," Proceedings of 10th IEEE Vehicular Networking Conference (VNC 2018), Taipei, Taiwan, December 2018.

[9] M. Uysal, Z. Ghassemlooy, A. Bekkali, A. Kadri and H. Menouar, "Visible Light Communication for Vehicular Networking: Performance Study of a V2V System Using a Measured Headlamp Beam Pattern Model," in IEEE Vehicular Technology Magazine, vol. 10, no. 4, pp. 45-53, Dec. 2015, doi: 10.1109/MVT.2015.2481561.

[10] Haas, H., Yin, L., Wang, Y. and Chen, C. (2015). What is lifi? Journal of lightwave technology, 34(6), 1533-1544.

[11] Haas, H. (2016, October). LiFi: Conceptions, misconceptions and opportunities. In 2016 IEEE Photonics Conference (IPC) (pp. 680681). IEEE.

[12] C. Tebruegge, A. Memedi, and F. Dressler, "Empirical Characterization of the NLOS Component for Vehicular Visible Light Communication," in 11th IEEE Vehicular Networking Conference (VNC 2019), Los Angeles, CA: IEEE, Dec. 2019, pp. 64-67.

[13] S. Bechadergue, H. Guan, L. Chassagne, S. TohmØ, and J.-L. Franchineau, "Visible light communication system for platooning applications," in Proc. Vehicle Infrastruct. Safety Improvement Adverse Conditions Night Driving, Oct. 2016.

[14] L. Cheng, W. Viriyasitavat, M. Boban and H. Tsai, "Comparison of Radio Frequency and Visible Light Propagation Channels for Vehicular Communications," in IEEE Access, vol. 6, pp. 2634-2644, 2018, doi: 10.1109/ACCESS.2017.2784620.

[15] M. Segata et al., "Towards inter-vehicle communication strategies for platooning support," 2014 7th International Workshop on Communication Technologies for Vehicles (Nets4Cars-Fall), St. Petersburg, Russia, 2014, pp. 1-6, doi: 10.1109/Nets4CarsFall.2014.7000903.

[16] "IEEE Standard for Local and metropolitan area networks - Part 15.7: Short-Range Wireless Optical Communication Using Visible Light," IEEE, Std 802.15.7-2011, Sep. 2011

[17] A.-M. Cailean and M. Dimian, "Impact of IEEE 802.15.7 Standard on Visible Light Communications Usage in Automotive Applications," IEEE Communications Magazine (COMMAG), 2017.

[18] Yacheur, B. Y., Ahmed, T., \& Mosbah, M. (2020, November). Analysis and Comparison of IEEE $802.11 \mathrm{p}$ and IEEE $802.11 \mathrm{bd}$. In International Workshop on Communication Technologies for Vehicles (pp. 55-65). Springer, Cham.

[19] H. Fernandez, L. Rubio, V. M. Rodrigo-Peñarrocha and J. Reig, "Path Loss Characterization for Vehicular Communications at $700 \mathrm{MHz}$ and 5.9 GHz Under LOS and NLOS Conditions," in IEEE Antennas and Wireless Propagation Letters, vol. 13, pp. 931-934, 2014, doi: 10.1109/LAWP.2014.2322261.

[20] Chandrasekharamenon, N. P., \& AnchareV, B. (2012). Connectivity analysis of one-dimensional vehicular ad hoc networks in fading channels. EURASIP Journal on Wireless Communications and Networking, 2012(1), 1-16. 